\title{
50 dB Parametric Gain in Silicon Photonic Wires
}

\author{
Bart Kuyken $^{1, \dagger}$, Xiaoping Liu ${ }^{2, \neq, \dagger}$, Günther Roelkens ${ }^{1}$, Roel Baets ${ }^{1}$, Richard M. Osgood Jr. ${ }^{2}$, and William M. J. Green ${ }^{3, *}$ \\ ${ }^{1}$ Photonics Research Group, Department of Information Technology, Ghent University - imec, Ghent B-9000, Belgium \\ ${ }^{2}$ Department of Electrical Engineering, Columbia University, 1300 S. W. Mudd Building, 500 W. $120^{\text {th }}$ Street, New York, NY 10027, USA \\ ${ }^{3}$ IBM Thomas J. Watson Research Center, 1101 Kitchawan Road, Yorktown Heights, NY 10598, USA \\ ${ }^{\ddagger}$ Current address:OFS Labs, 19 Schoolhouse Road, Somerset, NJ 08873, USA \\ ${ }^{\dagger}$ These authors contributed equally to this work. \\ *Corresponding author: wgreen@us.ibm.com
}

Received Month X, XXXX; revised Month X, XXXX; accepted Month X,

XXXX; posted Month X, XXXX (Doc. ID XXXXX); published Month X, XXXX

\begin{abstract}
A pulsed mid-infrared pump at $\lambda=2173 \mathrm{~nm}$ is used to demonstrate wideband optical parametric gain in a low-loss 2-cm long silicon photonic wire. Using dispersion engineering to obtain negative second-order $\left(\beta_{2}\right)$ and positive fourth-order $\left(\beta_{4}\right)$ dispersion, we generate broadband modulation instability and parametric fluorescence extending from $1911 \mathrm{~nm}-2486 \mathrm{~nm}$. Using a cw probe signal to interrogate the modulation instability spectrum, we demonstrate parametric amplification $>40 \mathrm{~dB}$ with an on-chip gain bandwidth wider than 580 nm, as well as narrowband Raman-assisted peak gain > 50 dB. (C) 2011 Optical Society of America

OCIS Codes: 130.4310, 190.4380, 190.4970
\end{abstract}

Two-photon absorption (TPA) in $\mathrm{Si}$ vanishes at wavelengths approaching $\lambda=2200 \mathrm{~nm}$, while its nonlinear Kerr refractive index $n_{2}$ stays comparatively constant $[1,2]$. Hence, the nonlinear figure of merit (FOM) $(n / \beta \lambda)$ increases dramatically near silicon's TPA threshold. The large intrinsic nonlinearity of $\mathrm{Si}$, when patterned into high-index-contrast dispersion-engineered silicon photonic wires, produces an ideal platform for the exploration of highly efficient, broadband, coherent nonlinear optical processes $[3,4]$. This platform can serve as an ideal host for chip-scale mid-IR applications [5-8] including molecular spectroscopy, free-space communication, and environmental monitoring.

Previously we reported a mid-IR optical parametric amplifier (OPA) with on-chip gain over a bandwidth of $220 \mathrm{~nm}$ in a 4-mm long silicon wire [5]. Using an improved Si wire design, we demonstrate here broadband mid-IR modulation instability (MI), having a bandwidth greater than $580 \mathrm{~nm}$ centered at a $2173 \mathrm{~nm}$ pump wavelength. The intense MI spectrum correlates with unprecedented values of on-chip parametric gain, exceeding $40 \mathrm{~dB}$. Moreover, we demonstrate that on-chip gain can exceed $50 \mathrm{~dB}$ in narrow Raman-scatteringassisted bands.

Our silicon wire is fabricated on a 200 mm silicon-oninsulator (SOI) wafer in a CMOS pilot line and is $2 \mathrm{~cm}$ long, with cross-sectional dimensions of $900 \mathrm{~nm}$ x $220 \mathrm{~nm}$ (inset Fig. 1(a)). The top and bottom cladding consist of air and a $2 \mu \mathrm{m}$ buried oxide (BOX), respectively. The waveguide operates in the fundamental quasi-TE mode, and has propagation losses of $<2.8 \mathrm{~dB} / \mathrm{cm}$ for $\lambda=2000$ $2500 \mathrm{~nm}$. These engineered waveguide dimensions produce a waveguide effective nonlinear parameter $\gamma \sim$ $130 \mathrm{~W}^{-1} \mathrm{~m}^{-1}$ and anomalous dispersion conditions $\left(\beta_{2}<0\right)$ at wavelengths from $1800-2400 \mathrm{~nm}$, as plotted in Fig. 1(a). Furthermore, $4^{\text {th-order }}$ dispersion is small and positive $\left(\beta_{4}>0\right)$ within the same wavelength range, which is needed for achieving broadband phase matching.

To achieve an efficient degenerate four-wave-mixing (FWM) process, i.e. two pump photons converted into one signal and one idler photon, $2 \omega_{p}=\omega_{s}+\omega_{i}$, the phase
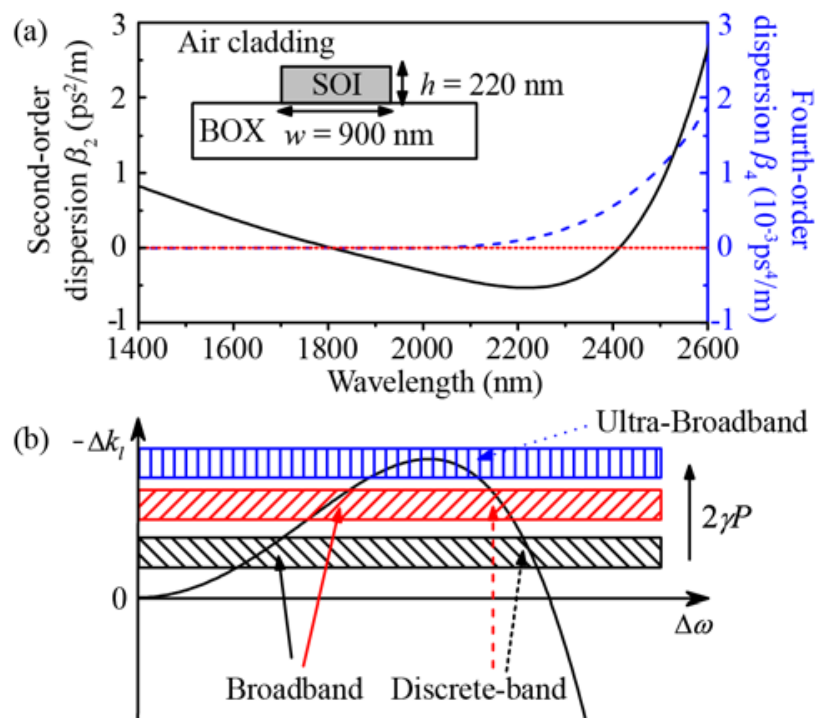

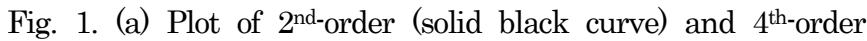
(dashed blue curve) dispersion for the fundamental quasi-TE mode of the silicon wire design shown in the inset. (b) Schematic illustration of pump-detuning-dependent broadband, discreteband, and ultra-broadband phase-matched solutions for FWM.

matching condition [9] restricts the frequency detuning of signal/idler from pump $\Delta \omega=\left|\omega_{p}-\omega_{s}\right|=\left|\omega_{p}-\omega_{i}\right|$ to values that satisfy $\left|\Delta k_{l}+2 \gamma P\right| \leq 2 \pi / L$ or $2 \gamma P-2 \pi / L \leq-\Delta k_{l}$ $\leq 2 \gamma P+2 \pi / L$, where $\Delta k_{l}$ is the linear phase-mismatch in propagation constants between signal/idler and pump, $\Delta k_{I}$ $=\left(k_{s}+k_{i}\right)-2 k_{p}, P$ is the input pump peak power and $L$ is the waveguide length. Graphically, when the $-\Delta k_{l}$ curve falls into a band between $2 \gamma P-2 \pi / L$ and $2 \gamma P+2 \pi / L$ (i.e. the shadowed bands in Fig. 1(b)), efficient phase matching results. Using a Taylor-series, $-\Delta k l$ can be related to $\Delta \omega$ through $-\Delta k_{l} \approx-\beta_{2}(\Delta \omega)^{2}-\beta_{4}(\Delta \omega)^{4 / 12}$. A typical representation of such a relation is shown as the black curve in Fig. 1(b). Clearly such a relation initially increases, then peaks, and decreases under the conditions $\beta_{2}<0 \& \beta_{4}>0$. At low power levels, there exist two phasematched bands, (shown by a solid black and dashed black 
arrow). For small frequency detuning, a region typically seen in parametric amplification [3, 4], the gain is broadband; at large frequency detuning, the gain has a narrower bandwidth, termed here a "discrete"-band [1012]. In addition, with increasing power level, the broadband phase-matched band and the discrete band shift away from and closer to the pump, respectively, until at high power levels, these two bands eventually merge together to create an even broader phase-matched band; this ultra-broadband is shown with the dotted blue arrow.

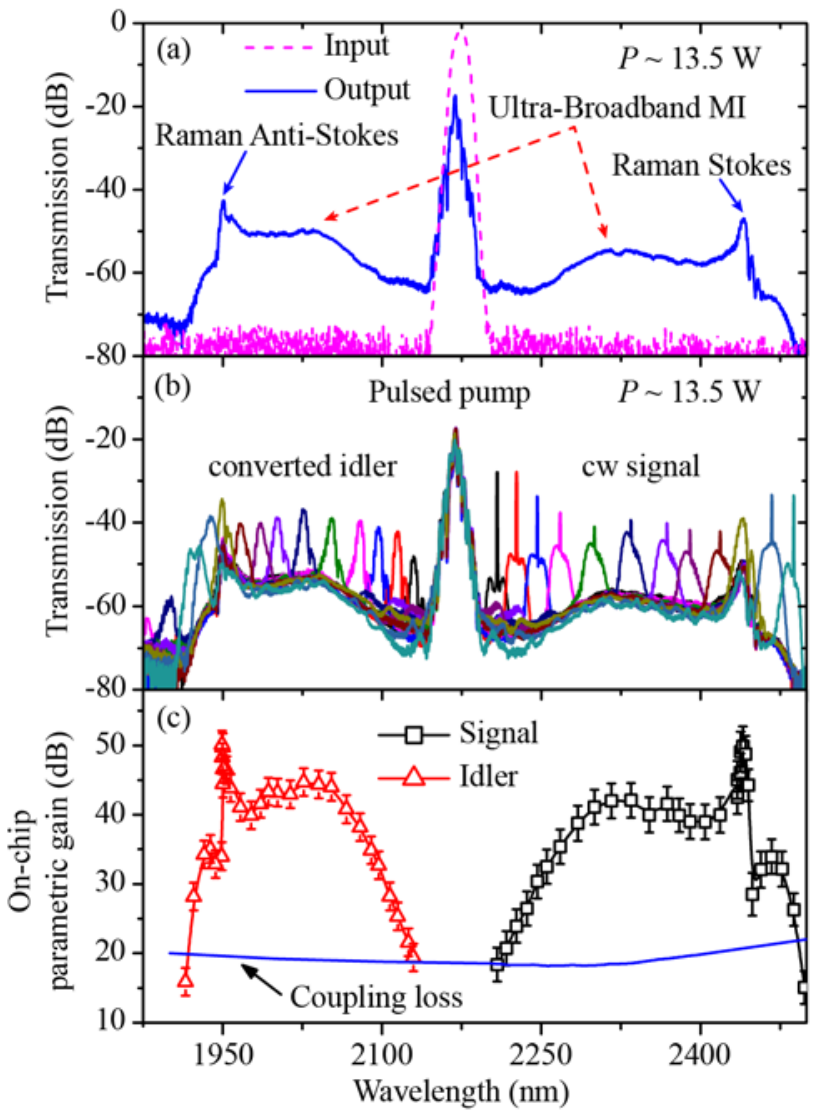

Fig. 2. (a) Input (dashed magenta) and output (solid blue) pump spectrum, taken with an input peak power $P \sim 13.5 \mathrm{~W}$ and $\lambda=$ $2173 \mathrm{~nm}$, illustrating ultra-broadband MI, and Raman Stokes/anti-Stokes peaks. (b) Series of FWM spectra with the pulsed pump co-propagating with a $\mathrm{cw}$ mid-IR signal at various wavelengths. The pulsed pump is centered at $\lambda=2173 \mathrm{~nm}$, and has $P \sim 13.5 \mathrm{~W}$. (c) Spectrum of on-chip parametric signal gain (black squares) and idler conversion gain (red triangles). Fiberwaveguide coupling loss is shown by the blue trace.

We performed a series of FWM experiments to investigate the bandwidth and magnitude of the parametric gain and wavelength conversion when operating within the ultra-broadband phase-matching region. The $\mathrm{Si}$ wire is pumped with a picosecond pulse train $(\mathrm{FWHM} \sim 2 \mathrm{ps}$, repetition rate $=76 \mathrm{MHz})$ from an OPA, operating at $\lambda=2173 \mathrm{~nm}$. The probe signal comes from a cw tunable mid-IR laser. The polarization of pump and probe are individually controlled to excite the $\mathrm{Si}$ wire's quasi-TE mode using in-line fiber polarization controllers. Pump and probe are multiplexed with a 90/10 single-mode fused-fiber coupler. Coupling into and out of the $2 \mathrm{~cm}$-long silicon wire is via tapered lensed fibers, with coupling losses of $\sim 10 \mathrm{~dB} /$ facet. An optical spectrum analyzer (OSA) is used to record the input and output transmission spectra at $1 \mathrm{~nm}$ resolution bandwidth.

The dashed magenta trace in Fig. 2(a) depicts the pulsed pump spectrum at the input of the photonic wire. The pump has an instrumentation-limited signal-to-noise ratio larger than $75 \mathrm{~dB}$, and a peak on-chip power of $P \sim$ $13.5 \mathrm{~W}$. The spectrum obtained at the wire output after transmission of the pump (solid blue curve in Fig. 2(a)) exhibits two features commonly observed for pulse propagation through nonlinear waveguides, these being self-phase modulation-induced (SPM) spectral oscillations on the original pump spectrum, as well as a spectral blueshift due to residual free-carrier dispersion [13]. Moreover, the transmitted spectrum exhibits additional two substantial modifications, each of which has been observed for the first time in silicon photonic wires. Most notably, the output spectrum is characterized by the emergence of a strong ultra-broadband modulation instability (MI) background [9, 14], having a bandwidth extending from $1911 \mathrm{~nm}$ to $2486 \mathrm{~nm}$. Furthermore, a prominent Raman Stokes peak, down-shifted from the pump by $\sim 15.6 \mathrm{THz}$ [8], rides on top of the MI spectrum at $\lambda=2411 \mathrm{~nm}$. Finally, a coherent Raman anti-Stokes [15] peak is visible at a wavelength of $1950 \mathrm{~nm}$. Sharp interference fringes occur near the Raman peaks, where the dispersion and phase shift due to the Raman susceptibility disrupts the broadband FWM phasematching condition [16].

The visibility of a strong MI background suggests that the on-chip mid-IR parametric gain available here is far larger than that demonstrated in previous studies [5], where MI was not observed. As shown by the overlaid spectra in Fig. 2(b), the parametric amplification is probed using a cw signal with wavelengths varying from $2209 \mathrm{~nm}$ - $2498 \mathrm{~nm}$ (8 nm step size) and generating the corresponding idler terms from $2129 \mathrm{~nm}-1914 \mathrm{~nm}$. The cw signal power coupled into the waveguide is kept low $(<$ $0.06 \mathrm{~mW}$ ) to prevent pump depletion, yet high enough to achieve visibility of the idler above the MI spectrum. Fig. 2(c) plots the measured on-chip amplification and conversion gain, evaluated following the approach in [5]. The mid-IR-pumped silicon wire OPA exhibits on-chip optical parametric amplification over a bandwidth exceeding $580 \mathrm{~nm}$. Within the Raman Stokes/anti-Stokes bands, the OPA reaches a maximum value of Ramanassisted parametric signal/idler gain of $\sim 50 \mathrm{~dB}$. Note that the gain profile also contains interference patterns near the Raman-assisted peaks, at wavelengths of $\sim 2445 \mathrm{~nm}$ and $\sim 1950 \mathrm{~nm}$; these features are related to those seen in the MI spectrum of Fig. 2(a). However, the number of interference fringes observed in Fig. 2(c) is reduced, because the cw probe's linewidth is broadened by crossphase modulation [13], in combination with the limited resolution of the 8-nm probe tuning step. After compensating for all fiber-chip coupling losses $(\sim 20 \mathrm{~dB})$, the OPA shows a net off-chip gain bandwidth of $\sim 550 \mathrm{~nm}$, with $~ 30 \mathrm{~dB}$ Raman-assisted peak off-chip gain for both signal and idler. The off-chip parametric amplification resulting solely from FWM can be $>20 \mathrm{~dB}$.

Large values of on-chip parametric amplification can lead to pump depletion [9] and even gain saturation at 
high input signal power; this is seen in a plot of output pulsed signal/idler peak power v.s. input cw signal power shown in Fig. 3(a). Here the input pump peak power $P \sim$ 13.5 W; the signal wavelength is near the FWM parametric gain maximum $\left(\lambda_{\mathrm{s}}=2356 \mathrm{~nm}\right)$, but outside the Raman band. At low signal-power levels, the power of the amplified signal and converted idler increase linearly with signal power, resulting in a signal and idler on-chip parametric gain of 39.5 and $42.3 \mathrm{~dB}$, respectively. The output signal/idler power saturates at $\sim 20 \mathrm{dBm}$, for signal powers $>-19 \mathrm{dBm}$. This gain saturation can be useful for limiting all-optical regeneration of signals [4], e.g. if the input signal is larger than $-19 \mathrm{dBm}$, the output signal is pinned at $\sim 20 \mathrm{dBm}$.
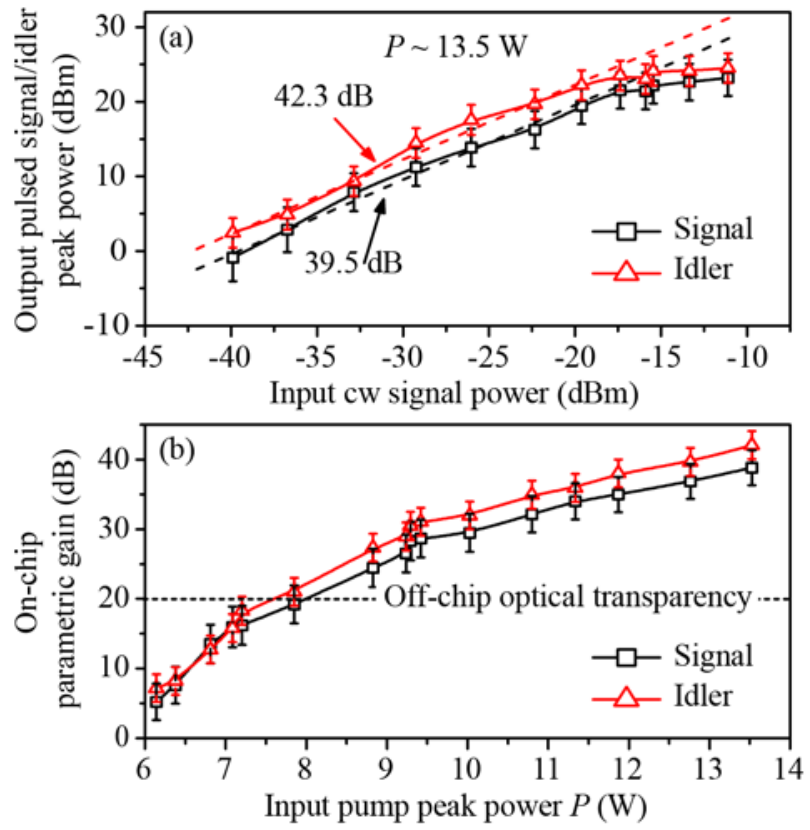

Fig. 3. (a) Output pulsed signal/idler peak power v.s. input cw signal power at $\lambda_{\mathrm{s}}=2356 \mathrm{~nm}$, with input pump peak power $P \sim$ $13.5 \mathrm{~W}$. (b) On-chip parametric gain as a function of $P$. Off-chip optical transparency is achieved with a peak power of $\sim 8 \mathrm{~W}$.

Fig. 3(b) plots the signal and idler on-chip parametric gain as a function of input peak pump power while keeping the signal wavelength at $\lambda_{\mathrm{s}}=2356 \mathrm{~nm}$ and its power at $-23.3 \mathrm{dBm}$. After compensating for the $20 \mathrm{~dB}$ coupling loss, off-chip optical transparency can be achieved for a peak pump power about $8 \mathrm{~W}$. Note that this can be further reduced with an improved coupling mechanism, i.e. a $\mathrm{SiO}_{\mathrm{x}} \mathrm{N}_{\mathrm{y}}$ coupler [17] instead of the cleaved facets used here. The differential gain is about $10.4 \mathrm{~dB} / \mathrm{W}$ at low peak power $\sim 6.5 \mathrm{~W}$ but decreases to 2.7 $\mathrm{dB} / \mathrm{W}$ for a peak power $>10 \mathrm{~W}$. This decrease is in part due to the residual nonlinear loss in the waveguide as discussed in [5, 18]. However as shown in Fig. 3(b) even at the highest peak power used in the experiments, the onchip parametric gain does not fully saturate, but instead continues to grow, but with a reduced differential gain.

The results obtained here represent a substantial improvement over those reported previously, for mid-IR parametric amplification in a $4 \mathrm{~mm}$-long silicon wire waveguide [5]. The peak operating pump power is reduced to less than half in the present device. At the same time, the maximum on-chip gain obtained using this $2 \mathrm{~cm}$-long wire here shows an improvement of more than $25 \mathrm{~dB}$, while the on-chip gain bandwidth is increased by more than $2.5 \times$. These results were achieved by leveraging judicious dispersion engineering and reduced propagation loss in the silicon wires, to achieve dramatic increases parametric gain and its bandwidth, including phasematching of the Raman scattering process

XL and RO would like to thank Sebastian Doetsch for many useful discussions during his stay in the US. XL thanks IBM for financial support, RO thanks NSF Award ID 0806682 for supporting this project. The authors also thank Yurii A. Vlasov at IBM Research for his support, and for many constructive discussions.

\section{References}

1. A. D. Bristow, N. Rotenberg, and H. M. van Driel, Appl. Phys. Lett. 90, 191104 (2007).

2. Q. Lin, J. Zhang, G. Piredda, R. W. Boyd, P. M. Fauchet, and G. P. Agrawal, Appl. Phys. Lett. 91, 021111 (2007).

3. M. A. Foster, A. C. Turner, J. E. Sharping, B. S. Schmidt, M. Lipson, and A. L. Gaeta, Nature 441, 960 (2006).

4. R. Salem, M. A. Foster, A. C. Turner, D. F. Geraghty, M. Lipson, and A. L. Gaeta, Nature Photonics 2, 35 (2008).

5. X. P. Liu, R. M. Osgood, Y. A. Vlasov, and W. M. J. Green, Nature Photonics 4, 557 (2010).

6. S. Zlatanovic, J. S. Park, S. Moro, J. M. C. Boggio, I. B. Divliansky, N. Alic, S. Mookherjea, and S. Radic, Nature Photonics 4, 561 (2010).

7. R. K. W. Lau, M. Menard, Y. Okawachi, M. A. Forst, A. C. Turner-Foster, R. Salem, M. Lipson, and A. L. Gaeta, Opt. Lett. 36, 1263 (2011).

8. V. Raghunathan, D. Borlaug, R. R. Rice, and B. Jalali, Opt. Express 15, 14355 (2007).

9. G. P. Agrawal, Nonlinear Fiber Optics (Academic, San Diego, 2001).

10. Q. Lin, T. J. Johnson, R. Perahia, C. P. Michael, and O. J. Painter, Opt. Express 16, 10596 (2008).

11. E. K. Tien, Y. W. Huang, S. M. Gao, Q. Song, F. Qian, S. K. Kalyoncu, and O. Boyraz, Opt. Express 18, 21981 (2010).

12.B. Kuyken, X. P. Liu, R. M. Osgood, Y. A. Vlasov, G. Roelkens, R. Baets, and W. M. J. Green, presented at OFC, Los Angeles, CA, USA, 2011, p. OTU4.

13. J. I. Dadap, N. C. Panoiu, X. G. Chen, I. W. Hsieh, X. P. Liu, C. Y. Chou, E. Dulkeith, S. J. McNab, F. N. Xia, W. M. J. Green, L. Sekaric, Y. A. Vlasov, and R. M. Osgood, Opt. Express 16, 1280 (2008).

14. N. C. Panoiu, X. F. Chen, and R. M. Osgood, Opt. Lett. 31, 3609 (2006).

15. R. Claps, V. Raghunathan, D. Dimitropoulos, and B. Jalali, Opt. Express 11, 2862 (2003).

16. M. R. E. Lamont, B. Luther-Davies, D. Y. Choi, S. Madden, X. Gai, and B. J. Eggleton, Opt. Express 16, 20374 (2008).

17. S. Assefa, C. Jahnes, and Y. Vlasov, US Patent US7738753B2 (2010).

18. S. Pearl, N. Rotenberg, and H. M. van Driel, Appl. Phys. Lett. 93, 131102 (2008). 